\title{
General Surgery in COVID: Challenges of a Government Sector Tertiary Care Medical University in a Developing Country and Lessons Learnt
}

\author{
Kushagra Gaurav ${ }^{1}$ Akshay Anand ${ }^{1, \odot}$ \\ Abhinav A. Sonkar ${ }^{1}$ \\ ${ }^{1}$ Department of General Surgery, King George's Medical University, \\ Lucknow, Uttar Pradesh, India
}

Int J Recent Surg Med Sci 2022;8:13-17.

\author{
Address for correspondence Manish K. Agrawal, MS, Department \\ of General Surgery, King George's Medical University, Lucknow \\ 226003, Uttar Pradesh, India \\ (e-mail: drmanishagrawal@rediffmail.com).
}

\begin{abstract}
Keywords

- general surgery

- COVID-19

- developing

- challenges

- government

Preserving resources and manpower is paramount in health care. It is important to ensure the ability of surgeons and specialized professionals to function through the pandemic. With one-sixth of world's population residing in a single developing country, with public health care expenditure being less than $1.28 \%$ of the total country's GDP and poor social protection, it seemed fighting a losing battle. However, a thoughtful and collaborative approach among leaders at every level has resulted in keeping the country afloat. The current coronavirus disease 2019 (COVID-19) pandemic underlines the importance of a mindful utilization of financial and human resources. Modifying international guidelines to fit the region-specific needs as per available resources is expected. This article is based on real-time challenges and experiences of a surgical department of a government medical university in planning and preparing clinical pathways during the COVID-19 pandemic to ensure high quality surgical care.
\end{abstract}

\section{Introduction}

The spread of coronavirus disease 2019 (COVID-19) in the entire globe has put the mankind at danger. Reports of the first COVID-19 cases started in December 2019 when Huang et al first recorded 27 cases of pneumonia of unexplained etiology in Wuhan, Hubei Province, People's Republic of China. ${ }^{1}$ Already COVID-19 epidemic is an international public health emergency and due to the rising severity of this recent outbreak, the finances of some of the biggest economies are stressed out.

\section{The Problem Statement: National Perspective}

The first case of COVID-19 with origin from China was registered in India on January 30, 2020. ${ }^{2}$ It has been well

DoI https://doi.org/ $10.1055 / \mathrm{s}-0041-1731980$ ISSN 2455-7420 understood that among various countries responding to the COVID-19 outbreak, India enforced one of the strongest lockdowns at an early phase of case growth-a score of 100 determined by an index created by the University of Oxford later. ${ }^{3}$ With the onset of the COVID-19 pandemic the Indian government has taken steps to avoid its spreadannouncing swiftly and strictly the lockdown measures on 22nd March 2020. ${ }^{4}$ Initial advisory from Ministry of Health and Family Welfare (MOHFW) suspended all air-traffic in last week of March and subsequently all nonessential operations were stopped while advising "social distancing" to be practiced as-"do gaj ki duri"-a distance of two yards. ${ }^{4-7}$ The whole idea of implementing world's first most significant lockdown was to give time for Indian health care to respond to gear up to fight such menace. With one-sixth of world's population residing in one country, the challenge was huge for the government agencies to handle. 
This lockdown went up to almost 3 months or so, but despite that community infections have been detected at multiple places and the outbreak curve did not flatten for a long time as it was expected. The nation cannot continue to permanently close off its economic operations/activities so gradual ease was allowed in lockdown and process of unlocking was started in a very slow and gradual manner. In addition to protecting the vulnerable, ramping up testing and public health infrastructure remained India's best way to take over the COVID marathon. COVID-19 has spread to almost across the length and breadth of the country. As per WHO, with end of February 2021 the total cases reported in India are about 1,11,12,241 confirmed cases of COVID-19 with $1,57,157$ deaths. ${ }^{8}$ Currently India ranks second among the

Table 1 Chronology of steps taken during the COVID-19 pandemic by the university

1. Nominating a senior physician as Nodal Officer and alerting infectious diseases unit (February 2020).

2. Starting of dedicated Fever Clinic on March 12th, 2020.

3. Proposing Digital OPD on March 15th, 2020 and limiting the outpatients clinics only for emergency services on March 18th, 2020.

4. Dedicated COVID-19 task force created; four specialized teams formed, viz., ventilatory ICU team, isolation team, triage team, and OPD team (each comprising of faculty and trainee residents), plan for training of each and every health care professional unrolled.

5. Statewide protocol for infection prevention and management of patients on nCOV released on March 20, 2020.

6. Creation of isolation/quarantine facilities and co-ordinating with State Department of Medical Education, Government of Uttar Pradesh India on daily basis from March 23, 2020.

7. Ventilatory training for all departments including surgery department from April 1, 2020.

8. Establishment of dedicated COVID-19 Surgical Task force on April 9, 2020.

9. Set-up of electronic COVID Care Network (eCCS)-to help other state medical colleges on May 8, 2020.

10. Starting semi-emergency services and ramping up existing emergency care from May 29, 2020.

11. Set up of COVID help desk in every building of the university-to help the poor patients coming to this tertiary care from June 26, 2020.

Abbreviations: COVID-19, coronavirus disease 2019; ICU, intensive care unit; OPD, outpatient department.
World and first in Asia in figures of total number of COVID 19 patients-after United States being top country with maximum numbers of patients. Despite this the country has registered one of the lowest mortality rates in the world.

\section{The Problem Statement: Institutional's Perspective}

King George's Medical University UP (KGMU UP) Lucknow India established more than 115 years ago is a tertiary health care center and a premier medical college and hospital catering to almost 6,000 patients on daily basis in outpatient clinic area, about $80 \%$ of whom are from below poverty line. Tackling COVID-19 in one of the busiest and largest British-era hospitals was a daunting task. The university administration answered the call immediately (-Table $\mathbf{1}$ ). Drawing evidence from across the country and around the world and implementing it is difficult in one go. With a flood of information pouring in from everywhere each day, designing and devising region-specific plans was a priority with the actual realization of the available resources. Creation of dedicated COVID-19 task force and activation of Infection Disease Group was the primary step. Agencies like Indian Council of Medical Research, Ministry of Health and Family Welfare (Govt. of India) and State Department of Medical Education, Government of Uttar Pradesh India issued regular updates and guidelines that resulted in subsequent changes in workforce management, staff allocation, day to day hospital operations, and clinical and academic schedules.

\section{The Problem Statement: Department's Perspective}

Department of General Surgery, KGMU, Lucknow, Uttar Pradesh, India is one of the oldest departments of the university ${ }^{9}$ with occupancy of 240 elective and 60 emergency beds. It has 23 faculty members fashioned in eight surgical units who practice general surgery besides multiple subspecialties-head and neck surgery, minimal invasive surgery, endocrine surgery, breast surgery, urology, vascular surgery, pulmonary surgery, surgical oncology, metabolic surgery, colorectal and hepatobiliary surgery. Department also contributes toward clinical and teaching responsibilities for 15 junior surgery residents admitted each year for a 3-year general surgical residency program with 15 senior residents admitted every year for training. Situated in the Northern

Table 2 Five years annual data of Department of Surgery, KGMU, UP India

\begin{tabular}{|l|l|l|l|l|l|l|}
\hline & $\mathbf{2 0 1 5 - 2 0 1 6}$ & $\mathbf{2 0 1 6 - 2 0 1 7}$ & $\mathbf{2 0 1 7 - 2 0 1 8}$ & $\mathbf{2 0 1 8 - 2 0 1 9}$ & $\mathbf{2 0 1 9 - 2 0 2 0}$ & $\mathbf{2 0 2 0 - 2 0 2 1}$ \\
\hline OPD & 50,859 & 50,445 & 54,299 & 65,737 & 69,541 & 14,869 \\
\hline Admissions & 10,831 & 7,263 & 6,621 & 8,184 & 8,789 & 3,973 \\
\hline $\begin{array}{l}\text { Major elective } \\
\text { surgeries }\end{array}$ & 4,308 & 4,381 & 4,446 & 4,450 & 4,657 & 1,065 \\
\hline $\begin{array}{l}\text { Minor elective } \\
\text { surgeries }\end{array}$ & 4,635 & 5,693 & 5,125 & 5,400 & 6,609 & 944 \\
\hline Emergency surgeries & 1,583 & 1,887 & 1,576 & 1,210 & 954 & 597 \\
\hline
\end{tabular}

Abbreviation: OPD, outpatient department. 
Indian heartland, this department in a 4,200 bedded medical university delivers primary to tertiary care to the region and adjoining countries including Nepal. - Table 2 summaries the annual data of the department for last 5 years (2015$2021)^{10}$ With this data we can infer that the department performs approximately 360 to 390 major and 390 to 550 minor surgeries (elective) and approximately 75 to 100 emergency surgeries every month. Ever since the pandemic broke up and lockdown was introduced in March 2020, the department saw a drastic fall of these numbers in 2020 (-Table 2).

Such downfall to almost nil routine elective surgeries was expected due to many reasons-first and foremost complete lockdown was introduced-only essential services (medical emergency facilities, basic amenities, etc.) were being carried out. Routine outpatient department (OPD) was shut down as per government advisory; only emergency consultations and surgical emergency procedures were performed with all necessary precautions. Public transport was completely stopped, and even strict cessation of private transport facility was also regularized. Secondly, the scarce availability of PPE (personal protective equipment) and lesser-known disease course resulted in accumulation of the undue fear of getting COVID-19 infection. This made both surgeons and patients to take only least possible elective and accept only emergency consultations. Though such drastic changes were also the need of the hour because in a developing country like India where there is already limited doctor to patient ratio and existing financial constraints, risking even a single doctor or any other HCP would amount to huge loss for future management. During the lockdown with time all the necessary arrangements were put in place by the state government advisory and e-consultations were started. However, since most of our patients are not educated and have no or limited access to internet services in the villages, the situation is going to be a real mayhem whenever all restrictions will be lifted.

With natural history and clinical presentation of the disease not so widely known and prevalent social and physical distancing measures, it was imperative to delay and postpone all nonemergency surgeries. A recent multi-institutional study spanning across continents estimated that based on a 12 -week average interruption of medical facilities due to COVID-19, about 28.4 million elective surgeries (72.3\%) worldwide will be canceled or delayed by the end of 2020. In India, according to the study estimation, over 5,00,000 patients may face surgical cancellations or delays over a 12 -week period around the time the novel coronavirus disease reaches its peak. ${ }^{11}$

\section{Challenges for General Surgeons: In a Developing Country Scenario}

The authors describe real-time experiences in planning and preparing clinical pathways developed and established in consultation with anesthesiology, intensive care medicine, microbiology and hospital administration during the COVID-19 pandemic to ensure high quality surgical care.
- In view of exponentially growing infection, $90 \%$ of wards were vacated and patients were discharged to ensure empty beds-all elective surgeries were suspended since March $24^{\text {th }}, 2020$ except cancer care.

- The British era hospital complex, most of which are 70 to 80 years old-areas were divided and designated for screening, triage, waiting area, isolation and quarantine zones.

- There was major shift in clinical and administrational responsibilities of the surgeons-most of them took the baton of designing and streamlining logistic institutional protocols for fighting COVID-19 in the university while others participated in COVID-19 patient care.

- The anesthesiologists and intensive care beds (ICBs) available for complicated surgical patients were redistributed, as they were designated for dedicated COVID-19 patients' care.

- The number of nursing staff, and other health care workers were also redistributed and organized into a central pool.

- All staff was specifically trained to don, doff, and dispose of PPEs including masks, eye protection, double nonsterile gloves, gowns, suites, caps, and socks through dedicated training modules arranged for them in local language. ${ }^{12}$

Certain protocols were designed especially for General Surgery in the university. Since the disease spreads through respiratory droplets, surgical and anesthesia teams are at highest risk due to high amount of aerosol generation during anesthesia and surgical procedures. American College of Surgeons (ACS), ${ }^{13}$ Society of American Gastrointestinal and Endoscopic Surgeons (SAGES), ${ }^{14}$ and Intercollegiate Royal College of Surgeons Guidance ${ }^{15}$ have separately designed protocols/guidelines for surgical care during this pandemic. Since there were no focused consensus guidelines from Indian subcontinent till June 2020, we designed few points based on our observations and logistics, inspired from above said guidelines.

\section{Prioritization of Surgical Interventions: "Surgical Triage"}

- Category A: Patients presenting with urgent and emergent surgical conditions were dealt with utmost priority and care. Clinical management was done as per standard treatment protocols for all surgical emergencies including no operational delay if required. All governmental and institutional guidelines were adhered to in terms of safety, active and passive quarantine by the HCPs involved in care.

- Category B: For patients known to be positive for COVID-19 or with high clinical suspicion of COVID-19 infection, nonoperational management was considered as clinically justified. This focus was granted from both medical and logistical standpoints. All decisions were discussed in a multidisciplinary meeting and were well documented.

- Category C: All elective surgical, laparoscopic, and endoscopic cases were postponed during this time (March to 
June 2020). However, all such decisions were individualized-for e.g., in unhealthy or symptomatic patient-the total risk of mortality and morbidity to the patient was assessed as an aggregate assessment of the real risk of proceeding versus delay of 6 to 8 weeks or more based on COVID-19 burden and in the context of medical, logistical, and organizational considerations.

\section{Establishment of Separate COVID-19 Operating Facility}

Operating cases with confirmed COVID-19 infections demand utmost safety for health care professionals (HCPs). The perioperative protocol is complex and is mainly suited for specialized designed "COVID operating room (ORs)." ${ }^{16} \mathrm{OR}$ design and flow are the key elements of quality assurance for ensuring minimal risk to HCP. This planning becomes more complex in a government scenario where cost constraints and manpower logistics are major issues. All these logistic and financial challenges were met with the setting up of a dedicated COVID-19 operating facility to deliver quality care without compromising the safety of patients as well as HCPs detailed as below:

- Treatment areas: that included emergency rooms, wards, ORs, and recovery rooms-were physically separated from each other keeping in mind the necessary fresh air changes. In COVID-19 ward/OR there was a separate entry and exit for HCPs and patients alike.

- Establishment of a surgical COVID-19 operating area: dedicated OR planning involved creation of OT task force committee involving the heads of various surgical branches and anesthesia. This team also required inputs from civil and electrical engineering personnel routinely since many structural and electrical changes in the existing system were needed. Like most of other places in the university, the building for surgery theatre was built in 1962, and the existing operating facility is not modular. Main challenges that were death with are as follow:
1. Choosing and modifying the OR: one OR with two separate exits was chosen. Gate A was chosen for entry of HCPs while Gate B was designated for entry and exit of patient. Ideally a negative pressured OR is recommended for COVID-19 surgeries, but since this facility was not available in the existing structure, four split air conditioning units and two exhaust fans were installed to create at least 25 air exchanges in the OR. ${ }^{17}$ HEPA units were installed at the exit of the exhaust fan ducts.

2. Defining separate donning and doffing area: preanesthetic room was designated as donning area. It was large enough to allow multiple people changing at a time but "buddy concept" was followed-two people change and enter at one time. When the first person dons, the other one acts as observer to guide him/her to maintain sterility of the PPE. With no separate doffing area-a small chamber was constructed with partitions on low cost basis-which included attached shower rooms separate for males and females HCPs. All doctors and nursing staff, OT technicians, and other members of the team were trained individually for ideal donning and doffing techniques.

3. Patient transport: Transport of the patients and connectivity to postoperative COVID-19 ward were made feasible with ease. OT area was cordoned off completely and entry and exit were guarded (-Fig. 1).

4. Waste disposal: Whole staff in the university was trained by microbiology department in collaboration with hospital waste management team for proper COVID waste disposal through both offline and online training modules multiple times. All the instruments were kept in hypochlorite solution (1\%) for about 20 minutes and then washed and autoclaved. Disposable linen and sheets were used and all these stuffs including PPEs were also kept in hypochlorite solution (1\%) for about 20 minutes and then sent for incineration through hospital waste management team.

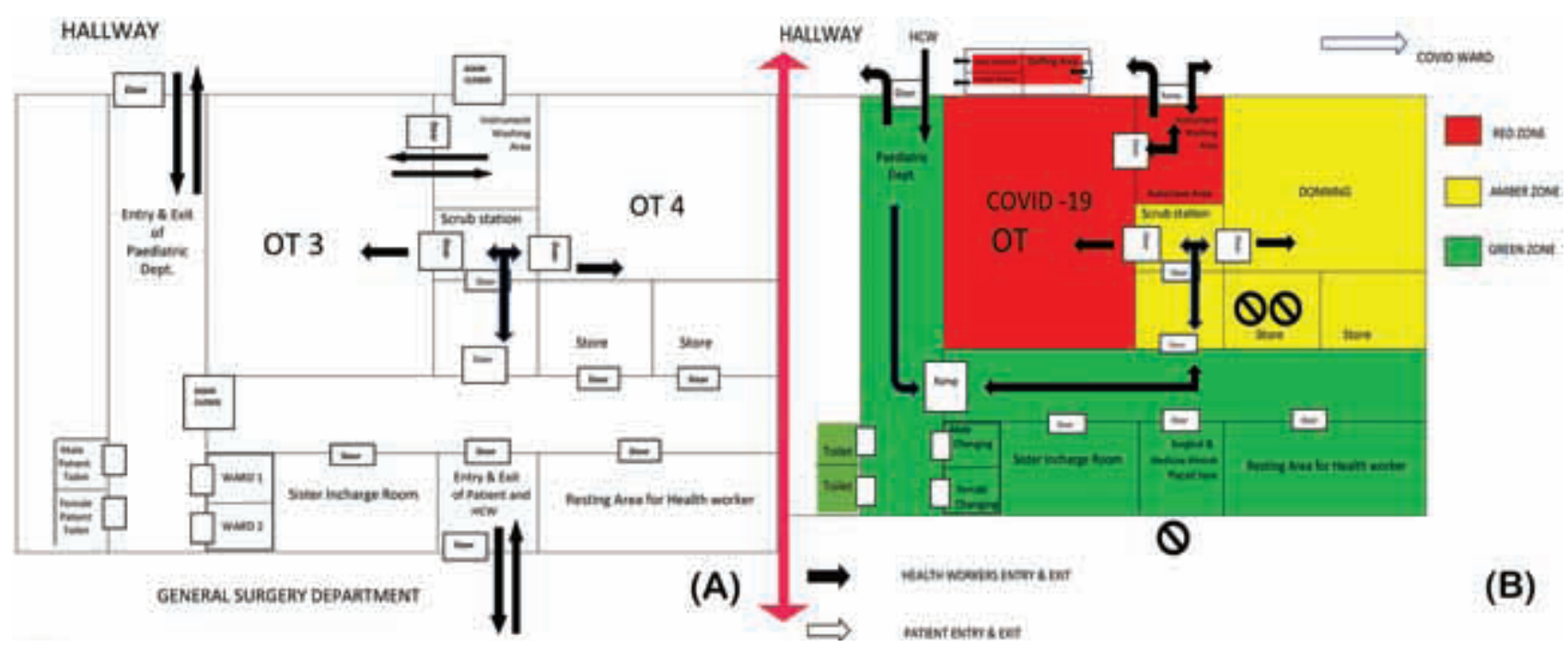

Fig. 1 Basic OR diagram. (A) Pre-COVID plan. (B) Post-COVID modifications showing red, amber, and green areas with separate patient and health care professional entry and exit plan. 
5. Recruiting and organizing manpower: shift duties were divided among clinicians, nurses, OT attendants, and members from transport and waste disposal so that every team was available round the clock. Similar back up team was also constituted for logistic reasons. Members of team were kept in active quarantine for 7 days, during which they participated in OR activities and then they were kept in passive quarantine for 14 days, being swabbed twice during that period.

\section{Lessons Learnt}

Country made specialized directives, early use of three ply masks, sanitizers, and educating all HCPs, use of tech-savvy WhatsApp communication and ramping testing frequency has proved to be game changers in pandemic management in our region. Dedicating one OR area for only COVID operative management for all surgical departments and developing treatment rooms near the same resulted in creating a safe work place considering our resources. Presently one of the major problems faced by the university is the quality of PPEs being supplied from various government agencies and nongovernment organizations. With the idea to boost the production from local manufacturers, the quality of PPE remains a vital thing to check on regular basis.

\section{Conclusion}

During times of crisis such as the COVID-19 pandemic, surgeons play a decisive role in delivering health care by leading from the front. Despite major overhauling and still delivering the results, surgeon is a key point man in developing and implementing these novel changes. Acknowledging limitations and devising plans around these roadblocks is the need of the hour to tackle this pandemic.

\section{Authors' Contributions}

K.G. and A.A. have shared co-first authorship of this paper since both have worked equally in conceptualizing the study, data analysis, preparation, and revising the manuscript.

\section{Conflict of Interest}

None declared.

\section{References}

1 Huang C, Wang Y, Li X, et al. Clinical features of patients infected with 2019 novel coronavirus in Wuhan, China. Lancet 2020;395(10223):497-506

2 Chakraborty I, Maity P. COVID-19 outbreak: migration, effects on society, global environment and prevention. Sci Total Environ 2020;728:138882

3 Accessed July 9, 2021 at https://www.bsg.ox.ac.uk/research/ research-projects/coronavirus-government-response-tracker

4 Accessed July 9, 2021 at https://ndma.gov.in/images/covid/ ndmaorder240320.pdf

5 Accessed July 9, 2021 at https://www.mohfw.gov.in/pdf/ Consolidated Traveladvisory Updated11032020.pdf

6 Accessed July 9, 2021 at https://www.mohfw.gov.in/pdf/ Traveladvisory.pdf

7 Accessed July 9, 2021 at https://dpe.gov.in/mha-guidelinesdated-14-04-2020

8 Accessed July 9, 2021 at https://covid19.who.int/region/searo/ country/in

9 Accessed July 9, 2021 at https://www.kgmu.org/department_ details.php?dept_id=31\&dept_type=2

10 Accessed July 9, 2021 at https://www.kgmu.org/department_details.php?dept_type=2\&dept_id=31\&page_type=pati ent_statistics

11 COVIDSurg Collaborative. Nepogodiev D, Bhangu A. Elective surgery cancellations due to the COVID-19 pandemic: global predictive modelling to inform surgical recovery plans. Br J Surg 2020;107(11):1440:1449

12 Accessed July 9, 2021 at https://www.kgmu.org/covid-19.php

13 Accessed July 9, 2021 at https://www.facs.org/covid-19/ clinical-guidance

14 Accessed July 9, 2021 at https://www.sages.org/ recommendations-surgical-response-covid-19/

15 Accessed July 9, 2021 at https://www.rcseng.ac.uk/ coronavirus/joint-guidance-for-surgeons-v2/

16 Flemming S, Hankir M, Ernestus RI, et al. Surgery in times of COVID-19-recommendations for hospital and patient management. Langenbecks Arch Surg 2020;405(3):359-364

17 Bhattacharya K. Are surgeons in India prepared for COVID-19? Indian J Surg 2020;1-2:1-2 